\title{
Errors in adult trauma resuscitation: a systematic review
}

\author{
Anton Nikouline $^{1}$ (1) $\cdot$ Andrew Quirion ${ }^{1} \cdot$ James J. Jung ${ }^{2,3} \cdot$ Brodie Nolan $^{1,3,4,5}$
}

Received: 15 October 2020 / Accepted: 18 March 2021 / Published online: 29 April 2021

(C) The Author(s), under exclusive licence to Canadian Association of Emergency Physicians (CAEP)/ Association Canadienne de Médecine d'Urgence (ACMU) 2021

\begin{abstract}
Introduction Trauma resuscitation at dedicated trauma centers typically consist of ad-hoc teams performing critical tasks in a time-limited manner. This creates a high stakes environment apt or avoidable errors. Reporting of errors in trauma resuscitation is generally center-dependent and lacks common terminology.

Methods We conducted a systematic review by searching Ovid Medline, Scopus and Embase from inception to February 24, 2021 for errors in adult trauma resuscitation. English studies published after 2001 were included. Studies were assessed by two independent reviewers for meeting inclusion/exclusion criteria. Errors were characterized from the included studies and a summary table was developed. Our review was prospectively registered with the International Prospective Register of Systematic Reviews (PROSPERO) (CRD42020152875).

Results The literature search retrieved 4658 articles with 26 meeting eligibility criteria. Errors were identified by morbidity and mortality rounds or other committee in $62 \%$, missed injuries on tertiary assessment or radiology review in $12 \%$, deviations from algorithmic guidelines in $12 \%$ or predefined for chest tube complications, critical incident reporting, aspiration or delays in care. In total there were 39 unique error types identified and divided into 9 categories including Emergency Medical Services handover, airway, assessment of injuries, patient monitoring and access, transfusion/blood related, management of injuries, team communication/dynamics, procedure error and disposition.

Conclusions Overall, our systematic review identified 39 unique error types in trauma resuscitation. Identifying these errors is imperative in developing systems for improvement of trauma care.
\end{abstract}

Keywords Trauma $\cdot$ Medical errors $\cdot$ Resuscitation $\cdot$ Critical Care $\cdot$ Emergency treatment

Anton Nikouline

anton.nikouline@mail.utoronto.ca

Andrew Quirion

andrew.quirion@mail.utoronto.ca

James J. Jung

jjung11@mgh.harvard.edu

Brodie Nolan

brodie.nolan@mail.utoronto.ca

1 Division of Emergency Medicine, Department of Medicine, University of Toronto, C. David Naylor Building, 6 Queen's Park Crescent West, Third Floor, Room 304, Toronto, ON M5S 3H2, Canada

2 Department of Surgery, Massachusetts General Hospital, Harvard Medical School, Boston, MA, USA

3 International Centre for Surgical Safety, St. Michael's Hospital, Toronto, ON, Canada

4 Li Ka Shing Knowledge Institute, St. Michael's Hospital, Toronto, ON, Canada

5 Department of Emergency Medicine, St. Michael's Hospital, Toronto, ON, Canada 


\section{Résumé}

Introduction La réanimation des traumatisés dans les centres de traumatologie spécialisés consiste généralement en des équipes ad hoc qui effectuent des tâches critiques dans un temps limité. Cela crée un environnement à enjeux élevés susceptible de provoquer des erreurs évitables. Le signalement des erreurs de réanimation traumatologique dépend généralement du centre et manque de terminologie commune

Méthodes Nous avons effectué une revue systématique en recherchant dans Ovid Medline, Scopus et Embase, du début au 24 février 2021, des erreurs de réanimation traumatologique chez l'adulte. Les études anglaises publiées après 2001 ont été incluses. Deux examinateurs indépendants ont évalué les études pour déterminer si elles répondaient aux critères d'inclusion et d'exclusion. Les erreurs ont été caractérisées à partir des études incluses et un tableau récapitulatif a été élaboré. Notre étude a été enregistrée de manière prospective dans l'International Prospective Register of Systematic Reviews (PROSPERO) (CRD42020152875).

Résultats La recherche documentaire a permis d'extraire 4658 articles, dont 26 répondaient aux critères d'admissibilité. Les erreurs ont été identifiées par les rondes de morbidité et de mortalité ou par un autre comité dans $62 \%$ des cas, les blessures manquées lors de l'évaluation tertiaire ou de l'examen radiologique dans $12 \%$ des cas, les déviations par rapport aux directives algorithmiques dans $12 \%$ des cas ou prédéfinies pour les complications du tube thoracique, le signalement des incidents critiques, l'aspiration ou les retards dans les soins. Au total, 39 types d'erreurs uniques ont été identifiés et répartis en 9 catégories : transfert des services médicaux d'urgence, voies respiratoires, évaluation des blessures, surveillance et accès du patient, transfusion/sang, gestion des blessures, communication/dynamique de l'équipe, erreur de procédure et disposition. Conclusions Dans l'ensemble, notre étude systématique a identifié 39 types d'erreurs uniques dans la réanimation des traumatismes. L'identification de ces erreurs est impérative pour développer des systèmes d'amélioration des soins en traumatologie.

\section{Clinician's capsule}

\section{What is known about the topic?}

There are a number of studies that identify errors in trauma but without common definitions or taxonomy.

\section{What did this study ask?}

What are the errors identified in current trauma resuscitation literature?

\section{What did this study find?}

The study found 39 unique error types in trauma resuscitation. These errors can be used to identify areas of improvement for trauma care.

\section{Why does this study matter to clinicians?}

This study identifies potential errors in trauma resuscitation which allows clinicians to be aware of errors during their trauma management and develop programs for error reduction for their institutions.

\section{Introduction}

Trauma care has seen a number of systems changes with centralizing trauma care into tertiary centers to provide specialized and expedited care[1-5]. Trauma resuscitation in these centers typically consist of ad-hoc teams performing critical tasks in a time limited manner[6, 7]. On average, a critical decision is required every $72 \mathrm{~s}$ [8]. This presents a unique high stakes environment that is apt for critical errors to occur in multiple facets of care.
A systematic review of preventable deaths in trauma found that inconsistent terms were used to describe errors in trauma [9]. Currently, reporting of errors in trauma are generally center-dependent and lack a common terminology [10]. Errors are commonly determined by mortality and morbidity rounds/ expert committee [10-15], benchmarking to accepted guidelines $[8,16,17]$ or by determining risk using severity scores (Injury Severity Score [ISS] or Trauma and Injury Severity Score [TRISS]) [10,11]. Amongst the different phases of trauma care, the initial trauma resuscitation phase presents the largest number of avoidable errors [11, 12, 14, 15].

Most recently, papers have been using the Joint Commission on Accreditation of Healthcare Organizations (JCAHO) taxonomy of errors to standardize reporting of preventable errors [18]. This classification system has been used and/or adapted in several studies across multiple medical specialties $[10,11,15]$.Currently, there is no standard model for classifying errors in trauma resuscitation. A better understanding of the types of errors present in the current literature is vital in developing a taxonomy for future studies.

The objective of this systematic review was to describe the errors that occur during trauma resuscitation. The following research question was formulated: "Among injured adults, what are the errors that occur during the acute trauma resuscitation?".

\section{Methods}

We performed a systematic review adhering to the standards of the Preferred Reporting Items for Systematic Review and Meta-Analysis Protocols (PRISMA-P) (Appendix A) [19] 
and registered with the International Prospective Register of Systematic Reviews (PROSPERO) (CRD42020152875). The systematic review was conducted and reported according to PRISMA statements [20]. Research ethics approval and informed consent were not required.

\section{Data sources and search strategy}

Ovid Medline, Scopus and Embase were searched from inception to February 24, 2021. Database-specific key index terms and text words related to error, adverse events, trauma, trauma bay, resuscitation and emergency department were used. Our search was limited to studies reported in English. Our detailed search strategy is presented in Appendix B. Reference lists of included studies were reviewed for eligibility. Studies were reviewed by a trauma expert for appropriateness. EndNote X7 (Reuters, New York) was used for reference management.

Eligibility Criteria.

Articles written in English and published between January 2001 and the search date February 24, 2021 were included. We included studies that examined resuscitations conducted by trauma teams, included patients age 16 years or older, and documented errors or adverse events in the ED or trauma bay. Studies were excluded if they focused on mental health trauma, included patients aged less than 16 , did not describe trauma care provided by trauma teams or did not specify type or location of errors.

\section{Study selection}

Titles were initially screened by one reviewer (AN). Abstract and full-text review were then conducted in duplicate by two independent reviewers (AN, AQ) with disagreements resolved by involvement of a third reviewer (BN). The list of included studies was verified by the third reviewer $(\mathrm{BN})$. Data extraction was done by two authors (AN,AQ) using Excel (Microsoft, WA).

\section{Assessment of bias}

Four items from the National Institutes of Health (NIH) Quality Assessment for Observational Cohort and Cross-Sectional Studies were used for the assessment of bias [21]. Studies were scored based on their rate of eligible participants, sampling bias, exposure measure bias and outcome measure bias. The modified method using only four items had been previously used by the authors and found to be adequate [22].

\section{Data synthesis}

The primary objective of the systematic review was to identify errors during trauma resuscitation. Errors were distilled from individual studies into a single list. These were then reviewed by two study investigators. Duplicate errors were combined where appropriate based on consensus and errors not applicable to the acute trauma resuscitation were removed. Themes derived from the initial list of errors were used to derive categories. The remaining errors were then divided into these categories.

\section{Results}

\section{Study selection}

The literature search retrieved 4658 publications. After duplicates were removed, 4070 articles remained. Five additional articles were identified through reference searches. Of the 4075 articles screened by title, 3933 were excluded. The remaining 142 abstracts were reviewed by two independent reviewers resulting in 93 abstracts being excluded. The remaining 49 publications were assessed in full. In total, 26 studies met the inclusion criteria and were included in the analysis of this systematic review. A flowchart of study selection is illustrated in Fig. 1.

\section{Study characteristics}

Table 1 demonstrates the study characteristics of the 26 included studies. Studies were primarily published in the USA (1026) but included Australia, Austria, Canada, China, Denmark, France, Germany, Netherlands, Spain, Sweden, Switzerland and the United Kingdom. Studies included were published from 2002 to 2020 . Seven (27\%) studies were conducted prospectively, eighteen (69\%) retrospectively and one (4\%) had both prospective and retrospective components. Errors were identified by morbidity and mortality rounds or other committee in 16 studies (62\%), missed injuries by computerized tomography (CT) scan/tertiary survey in three studies (12\%), deviation from a predefined algorithm based on guidelines in three studies (12\%) and the rest were specific to a critical incident reporting system (1), chest tube complications (1), aspiration event (1) or delays in care (1).

\section{Data sources and assessment of bias}

Appendix $\mathrm{C}$ demonstrates the different data sources and the number of errors that occurred during the resuscitation phase. Nineteen studies used chart review for data collection (73\%), five used direct observation (19\%) and two used video recording $(8 \%)$. The captured number of errors varied greatly amongst the studies ranging from 6 to 2689 with only seven studies having greater than 100 events $(27 \%)$. Overall bias was rated good (4/4) for 20 studies, fair (3/4) 
Fig. 1 Prisma flow diagram

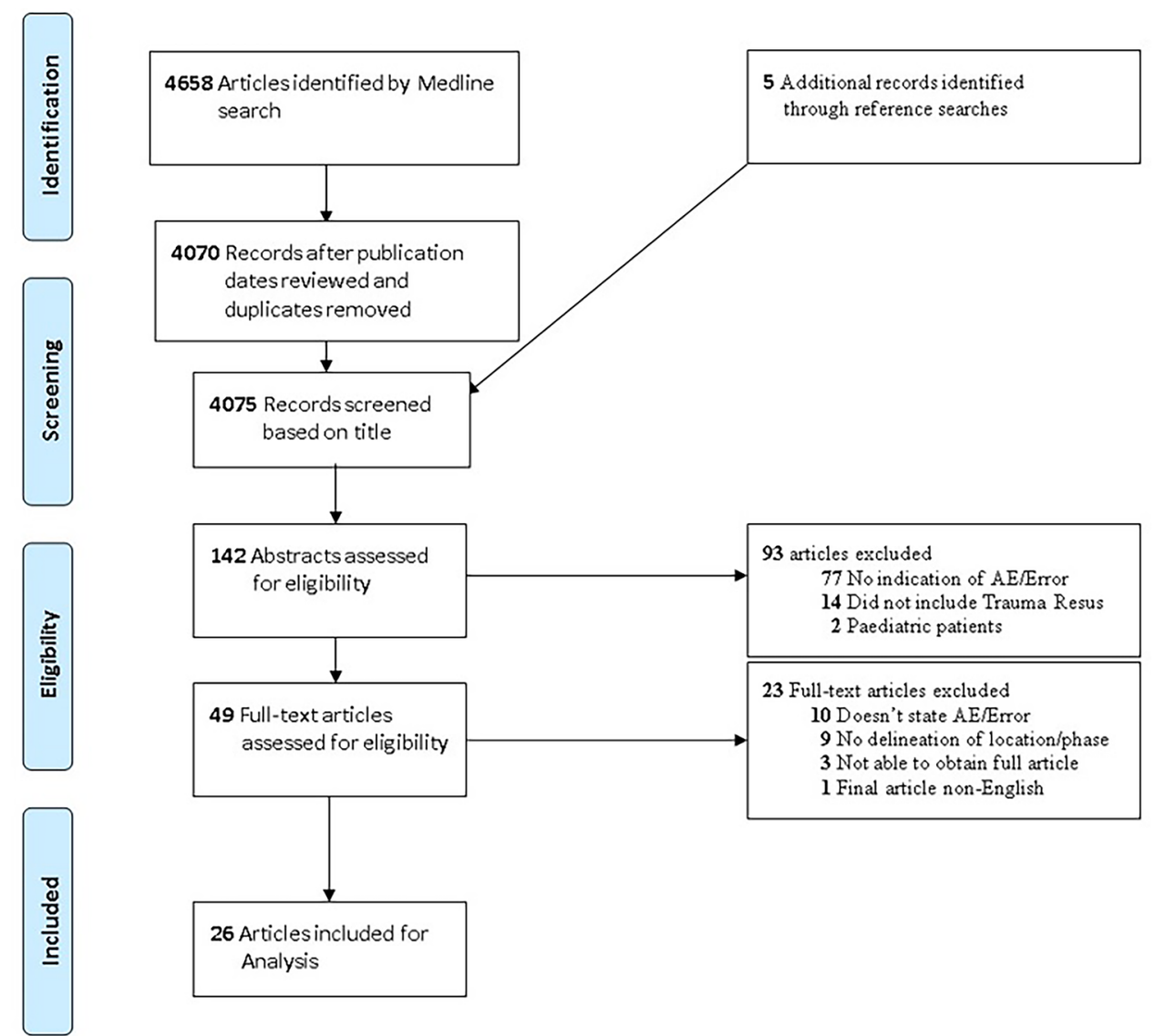

for 4 studies and two studies received a poor (1/4) rating for bias (Table 2).

\section{Summary of errors}

A full list of all 363 errors identified can be found in Appendix D. Two reviewers (BN, AN) individually removed duplicates and errors not applicable to trauma resuscitation. The two lists were then reviewed together and a total of 305 errors were either removed or amalgamated to create a list of 58 unique errors. Based on recurrent themes in the list, the remaining errors were categorized by stages of the trauma evaluation including Emergency Medical Services (EMS) handover, airway, assessment of injuries, patient monitoring and access, transfusion/blood related, management of injuries, team communication/dynamics, procedure error and disposition (Table 3). Once categorized, errors were further amalgamated upon review by two study investigators and a total of 39 error types were included and divided into 9 categories.

\section{Discussion}

\section{Interpretation of findings}

The identification of errors varied greatly among studies. Morbidity and mortality rounds have been shown to be limited by recall bias with inaccurate and insufficient details related to timeline, incidence and nature of errors [23]. The assessment by morbidity and mortality committees, although commonplace, is not a standardized practice between institutions and is a possible source of bias for error reporting. Development of standardized systems for identifying errors is imperative for improving trauma care. Sanddal et al. [13] standardized a system for review by presenting a checklist to their review panel including resource utilization, critical time intervals, cause of death and judgments on preventability and care. This system also determined opportunities of improvement regardless of whether the death was preventable or not. Similar systematic approaches would allow for broader and less biased identification of errors. A number of studies provided the distinction between active and latent failures as active failures contributing directly to adverse outcomes and latent failures as conditions predisposing to active failures [11, 12, 24]. The splitting of errors into active 


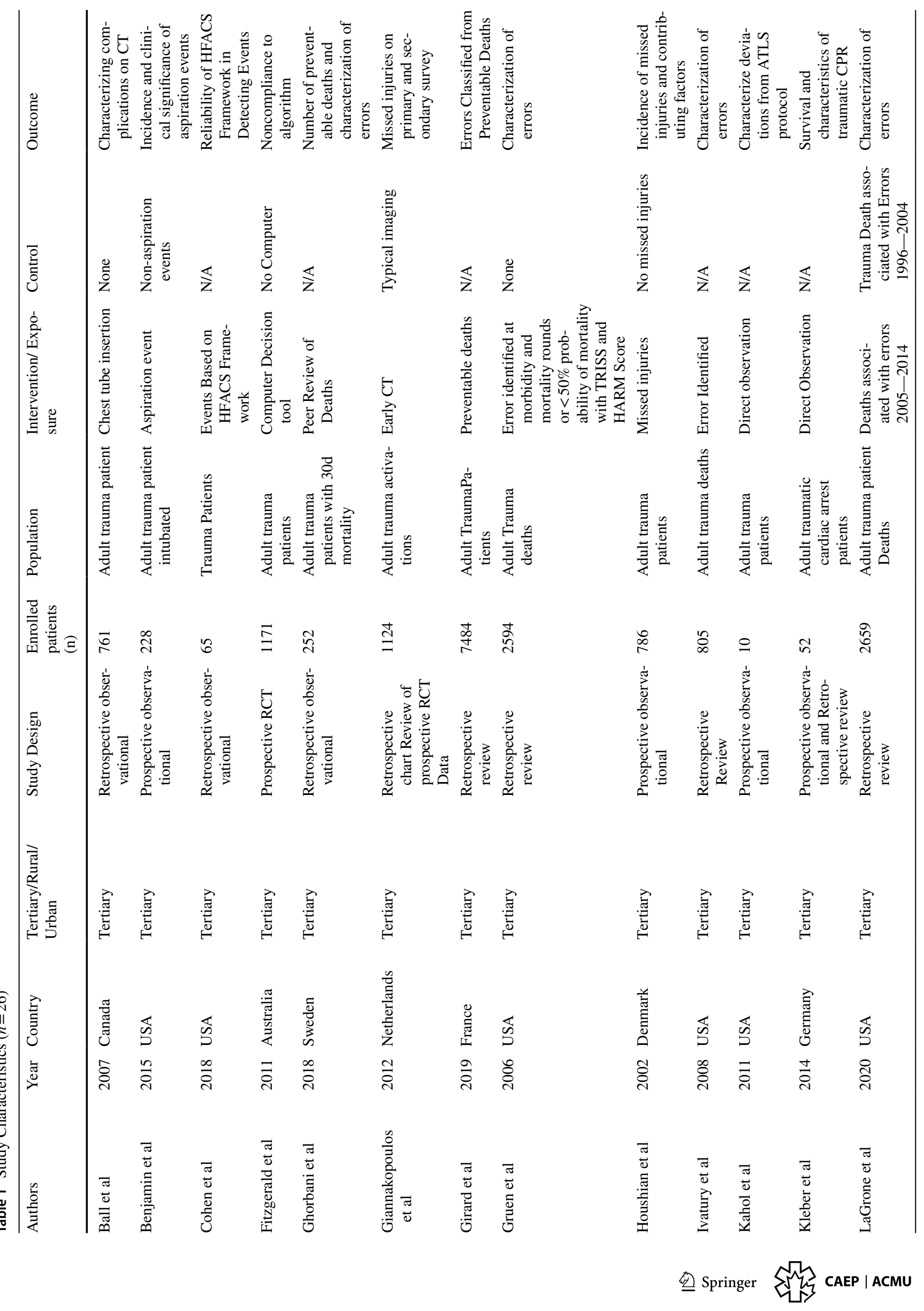




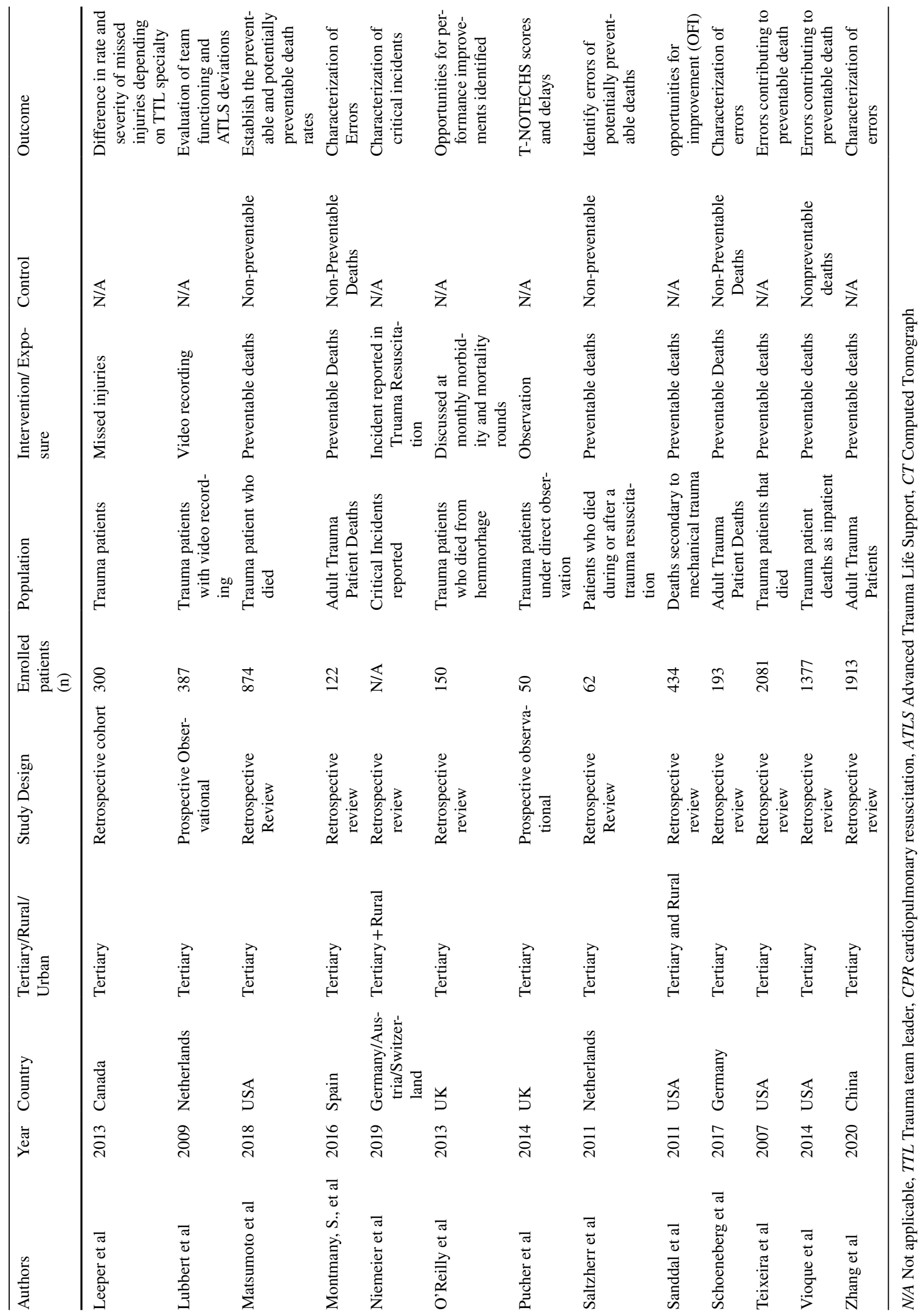


failures and latent failures is an important distinction when identifying and understanding cause. This distinction can help in developing solutions as active failures are more likely secondary to knowledge gaps and latent failures require more systematic solutions. LaGrone et al. [25] demonstrated the reduction in errors over two 10-year periods after instituting a number of protocols concerning trauma care targeting latent failures. Navarro et al. [26] consequently demonstrated a reduction of active failures through increased ATLS training for practitioners.

Three studies in our systematic review classified errors by injuries missed during the initial trauma resuscitation that were later identified. Giannakopoulos et al. [27] argue that according to Advanced Trauma Life Support (ATLS), more severe injuries should be prioritized and missed injuries aren't errors but rather a calculated risk. Gruen et al. [11] also reported a $2.47 \%$ death rate contributed to by error and suggested a $2-3 \%$ error-related death rate may be the baseline in complex trauma systems. An acceptable "miss rate" has yet to be determined but more standardized approaches to error identification could potentially lead to a higher error-related death rate or missed injuries.

Three studies in our systematic review detected errors by classifying deviations from an algorithm. Typically, these would be deviations from ATLS protocol, but it is important to note that tertiary trauma care centers may deviate from protocolized practice. Kahol et al. [17] acknowledged this aspect of care and classified deviations from ATLS as errors, innovations or procedural deviations that were either reactive or proactive based on expert review of direct observation cases. They found senior trainees more likely to perform innovations during resuscitation. This sort of acknowledgment of the fluid nature of trauma care also makes error identification difficult as some errors could be seen as innovations depending on the observer. Multiple factors need to be interpreted simultaneously to define errors in trauma resuscitation as the situation and priorities can quickly change but was not within the scope of our study.

Throughout our systematic review, errors were found to be more likely to occur in the trauma bay or emergency department and airway management $[28,29]$. This further emphasizes the importance of the proper identification of errors in trauma resuscitation and to target quality improvement towards these areas.

\section{Development of a trauma resuscitation error taxonomy}

Through this systematic review, we found that errors in trauma resuscitation were reported using a large array of terms and definitions. We were able to distill the terms down to a list of 39 errors found in trauma resuscitation. We summarized our findings in Table 3. Our categories were developed based on themes present in errors identified and closely follow ATLS protocol and the chronological approach to trauma resuscitation. We believe that this systematic review is the first step towards the development of a classification system for errors during trauma resuscitation. The JCAHO taxonomy is inadequate for trauma resuscitation due to the high number of errors exclusive to the initial resuscitation, the importance of the first hour in trauma care and the lack of specificity for this critical time period $[11,12,14,15]$. Adaptation of an agreed upon classification system for errors would allow for improved homogeneity of reporting in future studies and comparison between different centers [10]. A classification system could also be used for assessment of resuscitation by video review or machinelearning assisted algorithms to enhance patient safety in the trauma bay [30].

\section{Strengths and limitations}

The studies included in this systematic review were limited by their methodology. Many were retrospective analyses with small numbers of errors that likely limited the ability to determine severity. There was significant heterogeneity in how errors were reported and classified. The determination of many of the errors was based on committee review of mortality, which inherently biases publication of more severe errors and prevents the determination of non-lethal but important errors in trauma resuscitation. This systematic review was also limited to errors stated in literature during the search timeframe and it is likely that there are additional important events that should be included in any final taxonomy.

There was also great heterogeneity amongst studies based on country and trauma systems, where there may be variation of practice, emergency medical services protocols and access to care. This systematic review was limited to English studies. Studies that included direct observation may have been limited by the Hawthorne effect whereby people react differently upon being observed $[8,11]$.

\section{Clinical implications}

The first step to improvement is the recognition of errors. This systematic review presents a list of potential errors found in trauma resuscitation. Clinicians will be able to use this information to better analyze their own trauma management as well as develop quality improvement programs for their institutions. A number of studies have already demonstrated the benefit of simulation, multidisciplinary meetings, ATLS training and protocol development in reducing errors [26, 29, 31, 32]. 
Table 2 Risk of bias assessment

\begin{tabular}{|c|c|c|c|c|c|c|}
\hline Authors & Year & $\begin{array}{l}\text { Participa- } \\
\text { tion }>50 \%{ }^{a}\end{array}$ & Sampling bias ${ }^{\mathrm{b}}$ & $\begin{array}{l}\text { Exposure } \\
\text { measurement } \\
\text { bias }^{c}\end{array}$ & $\begin{array}{l}\text { Outcome } \\
\text { measurement } \\
\text { bias }^{d}\end{array}$ & $\begin{array}{l}\text { Overall } \\
\text { assessment }\end{array}$ \\
\hline Ball et al & 2007 & Yes & No & No & No & Good \\
\hline Benjamin et al & 2015 & Yes & No & No & No & Good \\
\hline Cohen et al & 2018 & Yes & Yes & Yes & Yes & Poor \\
\hline Fitzgerald et al & 2011 & Yes & Yes & No & No & Fair \\
\hline Ghorbani et al & 2018 & Yes & No & Yes & No & Fair \\
\hline Giannakopoulos et al & 2012 & Yes & No & No & No & Good \\
\hline Girard et al & 2019 & Yes & No & No & No & Good \\
\hline Gruen et al & 2006 & Yes & No & No & No & Good \\
\hline Houshian et al & 2002 & Yes & No & No & No & Good \\
\hline Ivatury et al & 2008 & Yes & No & No & No & Good \\
\hline Kahol et al & 2011 & Yes & No & No & No & Good \\
\hline Kleber et al & 2014 & Yes & No & No & No & Good \\
\hline LaGrone et al & 2020 & Yes & No & No & No & Good \\
\hline Leeper et al & 2013 & Yes & No & No & No & Good \\
\hline Lubbert et al & 2009 & Yes & No & Yes & No & Fair \\
\hline Matsumoto et al & 2018 & Yes & No & No & No & Good \\
\hline Montmany et al & 2016 & Yes & No & No & No & Good \\
\hline Niemeier et al & 2019 & Yes & No & No & No & Good \\
\hline O'Reilly et al & 2013 & Yes & No & No & No & Good \\
\hline Pucher et al & 2014 & Yes & Yes & Yes & Yes & Poor \\
\hline Saltzherr et al & 2011 & Yes & No & No & No & Good \\
\hline Sanddal et al & 2011 & Yes & No & No & No & Good \\
\hline Schoeneberg et al & 2017 & Yes & No & No & Yes & Fair \\
\hline Teixeira et al & 2007 & Yes & No & No & No & Good \\
\hline Vioque et al & 2014 & Yes & No & No & No & Good \\
\hline Zhang et al & 2020 & Yes & No & No & No & Good \\
\hline
\end{tabular}

${ }^{a}$ Was the participation rate of eligible persons at least $50 \%$ ?

${ }^{b}$ Were all the subjects selected or recruited from the same or similar populations (including the same time period)? Were inclusion and exclusion criteria for being in the study pre-specified and applied uniformly to all participants?

${ }^{c}$ Were the exposure measures (independent variables) clearly defined, valid, reliable, and implemented consistently across all participants?

${ }^{\mathrm{d}}$ Were the outcome measures (dependent variables) clearly defined, valid, reliable, and implemented consistently across all participants?

${ }^{e}$ Overall assessment $4 / 4=$ Good, $2-3 / 4=$ Fair, $0-1 / 4=$ Poor

\section{Research implications}

In developing a taxonomy, our list of identified errors would require further input from trauma care experts to assess if there are additional missing or relevant errors that were not found in the literature and would require appropriate validation studies. Further development could be done through expert consensus using techniques such as modified Delphi or RAND appropriateness methods [33]. Assignment of weight and importance to these errors would also be important in developing a taxonomy for real world application. Unfortunately our study was unable to determine severity based on frequency, as other studies, due to the heterogeneity of errors identified and the variable number of captured events between studies [34]. Currently, the Clavien-Dindo classification system is commonly used to grade severity of surgical complications [35]. Future studies could use a similar system to grade trauma errors with Grade I requiring no intervention, Grade II requiring pharmacological intervention, Grade III requiring procedural intervention, Grade IV causing a life threatening complication and Grade V causing death. This system could be easily applied to trauma but would need further characterization and validation. Ultimately a prospective systematic approach with well-established methodology, such as the Health Care Failure Mode and Effect Analysis ${ }^{\mathrm{TM}}$ method, 
Table 3 Errors identified and proposed classification system

\begin{tabular}{|c|c|c|}
\hline $\begin{array}{l}\text { EMS handover } \\
\text { Failure or delay to activate trauma } \\
\text { team } \\
\text { Inaccurate or incomplete medical } \\
\text { history report } \\
\text { Delay to arrival of trauma team/ } \\
\text { team member }\end{array}$ & $\begin{array}{l}\text { Inadequate monitoring } \\
\text { Equipment failure } \\
\text { Issues with IV access } \\
\text { Issues with bloodwork }\end{array}$ & $\begin{array}{l}\text { Team communication/dynamics } \\
\text { Communication error } \\
\text { Team member unavailable } \\
\text { Personnel issue } \\
\text { Ineffective team leadership }\end{array}$ \\
\hline $\begin{array}{l}\text { Airway } \\
\text { Hypoxia not addressed } \\
\text { Unanticipated loss of airway } \\
\text { Delay in intubation } \\
\text { Unsuccessful intubation attempt } \\
\text { Aspiration event } \\
\text { Ventilator malfunction }\end{array}$ & $\begin{array}{l}\text { Transfusion/blood } \\
\text { related } \\
\text { Delay to blood product } \\
\text { administration } \\
\text { Failure to follow } \\
\text { appropriate transfusion } \\
\text { protocol } \\
\text { Over-resuscitation with } \\
\text { crystalloid fluids } \\
\text { Inadequate blood therapy }\end{array}$ & $\begin{array}{l}\text { Procedure related } \\
\text { Technical errors } \\
\text { Equipment failure/missing } \\
\text { Procedure delayed } \\
\text { Procedure inappropriate (e.g., femoral } \\
\text { access in setting of intra-abdominal } \\
\text { bleeding) } \\
\text { Iatrogenic injury }\end{array}$ \\
\hline $\begin{array}{l}\text { Assessment of injuries } \\
\text { Missed/delayed identification of } \\
\text { injury/shock } \\
\text { Failure to follow cervical spine } \\
\text { precautions (if indicated) } \\
\text { Imaging not complete or delayed } \\
\text { Delay secondary assessment } \\
\text { Radiology misinterpreted } \\
\text { Inappropriate PPE }\end{array}$ & $\begin{array}{l}\text { Management of injuries } \\
\text { Failure or delay to appro- } \\
\text { priately treat severe } \\
\text { injury } \\
\text { Incorrect/ineffective } \\
\text { treatment of severe } \\
\text { injury } \\
\text { Complications of an } \\
\text { appropriate treatment } \\
\text { Medication error } \\
\text { Inadequate temperature } \\
\text { management }\end{array}$ & $\begin{array}{l}\text { Disposition } \\
\text { Delay to CT/OR/angiography suite } \\
\text { Inappropriate transfer of unstable patient }\end{array}$ \\
\hline
\end{tabular}

$I V$ intravenous, $C T$ computed tomography, $O R$ operating room, $P P E$ personal protective equipment would be imperative in determining further errors and their impact on patient care [36].

\section{Conclusion}

Overall, our systematic review identified 39 unique error types in trauma resuscitation which were categorized by themes. This systematic review provides a first step towards the creation of a common classification system and evaluation of severity for errors during trauma resuscitation. Clinicians can use these identified errors to help recognize errors at their own institution and develop improvements to their trauma care programs.

Supplementary Information The online version contains supplementary material available at https://doi.org/10.1007/s43678-021-00118-7.

Acknowledgements The authors would like to thank David Lightfoot, information specialist at St. Michael's Hospital, for his assistance with the literature search and development of the search strategy.

Author contributions AN participated in the literature search, data collection, data analysis, data interpretation, study design and writing of the manuscript, AQ participated in the data collection and data analysis. JJ participated in the study design, data interpretation and critical revision. BN participated in the study design, data analysis, data interpretation, writing and critical revision.

Funding There was no specific funding used for this study from any agency in the public, commercial or not-for-profit sectors.

Availability of data and material Search strategy available in Appendix B.

\section{Declarations}

Conflict of interest None declared.

Ethics approval None required for systematic review.

\section{References}

1. Haas B, Stukel TA, Gomez D, Zagorski B, De Mestral C, Sharma $\mathrm{SV}$, et al. The mortality benefit of direct trauma center transport in a regional trauma system: a population-based analysis. J Trauma Acute Care Surg. 2012;72(6):1510-7.

2. Haas B, Jurkovich GJ, Wang J, Rivara FP, MacKenzie EJ, Nathens AB. Survival advantage in trauma centers: expeditious intervention or experience? J Am Coll Surg. 2009;208(1):28-36.

3. Nolan B, Tien H, Sawadsky B, Rizoli S, McFarlan A, Phillips A, et al. Comparison of helicopter emergency medical services transport types and delays on patient outcomes at two level I trauma centers. Prehospital Emerg Care. 2017;21(3):327-33. 
4. Davis JW, Hoyt DB, McArdle MS, Mackersie RC, Shackford SR, Eastman AB. The significance of critical care errors in causing preventable death in trauma patients in a trauma system. J Trauma - Inj Infect Crit Care. 1991;31(6):813-9.

5. Shackford SR, Hollingworth-Fridlund P, Cooper GF, Eastman $\mathrm{AB}$. The effect of regionalization upon the quality of trauma care as assessed by concurrent audit before and after institution of a trauma system: a preliminary report. J Trauma - Inj Infect Crit Care. 1986;26(9):812-20.

6. Adedeji OA, Driscoll PA. The trauma team-a system of initial trauma care. Postgrad Med J. 1996;72:587-93.

7. Speck RM, Jones G, Barg FK, McCunn M. Team composition and perceived roles of team members in the trauma bay. J Trauma Nurs. 2012;19(3):133-8.

8. Fitzgerald M, Cameron P, Mackenzie C, Farrow N, Scicluna P, Gocentas R, et al. Trauma resuscitation errors and computerassisted decision support. Arch Surg. 2011;146(2):218-25.

9. Settervall CHC, Domingues C de A, de Sousa RMC, Nogueira L de S. Preventable trauma deaths, vol. 46, Revista de Saude Publica. Faculdade de Saúde Pública da Universidade de São Paulo; 2012. p. 367-75.

10. Vioque SM, Kim PK, McMaster J, Gallagher J, Allen SR, Holena DN, et al. Classifying errors in preventable and potentially preventable trauma deaths: A 9-year review using the Joint Commission's standardized methodology. Am J Surg. 2014;208(2):187-94.

11. Gruen RL, Jurkovich GJ, McIntyre LK, Foy HM, Maier RV. Patterns of errors contributing to trauma mortality: lessons learned from 2,594 deaths. Ann Surg. 2006;244(3):371-80.

12. O'Reilly D, Mahendran K, West A, Shirley P, Walsh M, Tai $\mathrm{N}$. Opportunities for improvement in the management of patients who die from haemorrhage after trauma. Br J Surg. 2013;100(6):749-55.

13. Sanddal TL, Esposito TJ, Whitney JR, Hartford D, Taillac PP, Mann NC, et al. Analysis of preventable trauma deaths and opportunities for trauma care improvement in utah. J Trauma. 2011;70(4):970-7.

14. Teixeira PG, Inaba K, Hadjizacharia P, Brown C, Salim A, Rhee P, et al. Preventable or potentially preventable mortality at a mature trauma center. J Trauma. 2007;63(6):1338-47.

15. Ivatury RR, Guilford K, Malhotra AK, Duane T, Aboutanos M, Martin N. Patient safety in trauma: Maximal impact management errors at a level I trauma center. J Trauma Inj Infect Crit Care. 2008;64(2):265-70.

16. The American College of Surgeons. Advanced trauma life support (ATLS ${ }^{\circledR)}$ Student Course Manual: the ninth edition. J Trauma Acute Care Surg. 2013;74(5):1363-6.

17. Kahol K, Vankipuram M, Patel VL, Smith ML. Deviations from protocol in a complex Trauma environment: Errors or innovations? J Biomed Inform. 2011;44(3):425-31.

18. Chang A, Schyve PM, Croteau RJ, O'leary DS, Loeb JM. The JCAHO patient safety event taxonomy: a standardized terminology and classification schema for near misses and adverse events. Int J Qual Heal Care. 2005;17(2):95-105.

19. Moher D, Shamseer L, Clarke M, Ghersi D, Liberati A, Petticrew $\mathrm{M}$, et al. Preferred reporting items for systematic review and metaanalysis protocols (PRISMA-P) 2015 statement. Rev Esp Nutr Humana y Diet. 2016;20(2):148-60.

20. Liberati A, Altman DG, Tetzlaff J, Mulrow C, Gøtzsche PC, Ioannidis JPA, et al. The PRISMA statement for reporting systematic reviews and meta-analyses of studies that evaluate health care interventions: explanation and elaboration. PLoS Med. 2009;6(7): https://doi.org/10.1371/journal.pmed.1000100.
21. National Institute of Health. National institue of health quality assessment tool for observational cohort and cross-sectional studies. 2017.

22. Jung JJ, Elfassy J, Jüni P, Grantcharov T. Adverse events in the operating room: definitions, prevalence, and characteristics. a systematic review. World J Surg. 2019;43:2379-92. https://doi.org/ 10.1007/s00268-019-05048-1.

23. Hutter MM, Rowell KS, Devaney LA, Sokal SM, Warshaw AL, Abbott WM, et al. Identification of surgical complications and deaths: an assessment of the traditional surgical morbidity and mortality conference compared with the american college of surgeons-national surgical quality improvement program. J Am Coll Surg. 2006;203(5):618-24.

24. Cohen TN, Cabrera JS, Litzinger TL, Captain KA, Fabian MA, Miles SG, et al. Proactive safety management in trauma care: applying the human factors analysis and classification system. J Healthc Qual. 2018;40(2):89-96.

25. LaGrone LN, McIntyre L, Riggle A, Robinson BRH, Maier RV, Bulger $\mathrm{E}$, et al. Changes in error patterns in unanticipated trauma deaths during 20 years: In pursuit of zero preventable deaths. J Trauma Acute Care Surg. 2020;89(6):1046-53.

26. Navarro S, Montmany S, Rebasa P, Colilles C, Pallisera A. Impact of ATLS training on preventable and potentially preventable deaths. World J Surg. 2014;38(9):2273-8.

27. Giannakopoulos GF, Saltzherr TP, Beenen LF, Reitsma JB, Bloemers FW, Goslings JC, et al. Missed injuries during the initial assessment in a cohort of 1124 level-1 trauma patients. Injury. 2012;43(9):1517-21.

28. Schoeneberg C, Schilling M, Hussmann B, Schmitz D, Lendemans S, Ruchholtz S. Preventable and potentially preventable deaths in severely injured patients: a retrospective analysis including patterns of errors. Eur J Trauma Emerg Surg. 2017;43(4):481-9.

29. Montmany S, Pallisera A, Rebasa P, Campos A, Colilles C, Luna A, et al. Preventable deaths and potentially preventable deaths. What are our errors? Injury. 2016;47(3):669-73.

30. Nolan B, Hicks C, Petrosoniak A, Jung J, Grantcharov T. Pushing boundaries of video review in trauma: using comprehensive data to improve the safety of trauma care. Trauma Surg Acute Care Open. 2020;5(1):e000510.

31. Long AM, Lefebvre CM, Masneri DA, Mowery NT, Chang MC, Johnson JE, et al. The golden opportunity: multidisciplinary simulation training improves trauma team efficiency. J Surg Educ. 2019;76(4):1116-21.

32. Girard E, Jegousso Q, Boussat B, Francois P, Ageron FX, Letoublon $\mathrm{C}$, et al. Preventable deaths in a French regional trauma system: a 6-year analysis of severe trauma mortality. J Visc Surg. 2019;156(1):10-6.

33. Campbell SM, Braspenning J, Hutchinson A, Marshall M. Research methods used in developing and applying quality indicators in primary care. Qual Saf Heal Care. 2002;11:358-64.

34. Fernald DH, Pace WD, Harris DM, West DR, Main DS, Westfall JM. Event reporting to a primary care patient safety reporting system: a report from the ASIPS collaborative. Ann Fam Med. 2004;2(4):327-32.

35. Bosma E, Veen EJ, Roukema JA. Incidence, nature and impact of error in surgery. Br J Surg. 2011;98(11):1654-9.

36. DeRosier J, Stalhandske E, Bagian JP, Nudell T. Using health care failure mode and effect analysis: the va national center for patient safety's prospective risk analysis system. Jt Comm J Qual Improv. 2002;28(5):248-67. 